\title{
Demonstrating Reduced-Voltage FPGA-Based Neural Network Acceleration for Power-Efficiency
}

\author{
Erhan Baturay Onural \\ TOBB ETÜ
}

\author{
Ismail Emir Yuksel \\ TOBB ETÜ
}

\author{
Behzad Salami \\ $B S C$
}

This demo aims to demonstrate undervolting below the nominal level set by the vendor for off-the-shelf FPGAs running Deep Neural Networks (DNNs), to achieve powerefficiency. FPGAs are becoming popular [1-4], thanks to their higher throughput than GPUs and better flexibility than ASICs. To further improve the power-efficiency, we propose to employ undervolting below the nominal level (i.e., $V_{\text {nom }}=$ $850 \mathrm{mV}$ for studied platform). FPGA vendors usually add a voltage guardband to ensure the correct operation under the worst-case circuit and environmental conditions [5-9]. However, these guardbands can be very conservative and unnecessary for state-of-the-art applications. Reducing the voltage in this guardband region does not lead to reliability issues under normal operating conditions, and thus, eliminating it can result in a significant power reduction for a wide variety of real-world applications. We will experimentally demonstrate a large voltage guardband for modern FPGAs: an average of 33\%. Eliminating this guardband leads to significant power-efficiency $(G O P s / W)$ improvement, on average, 2.6X, see Figure 1.

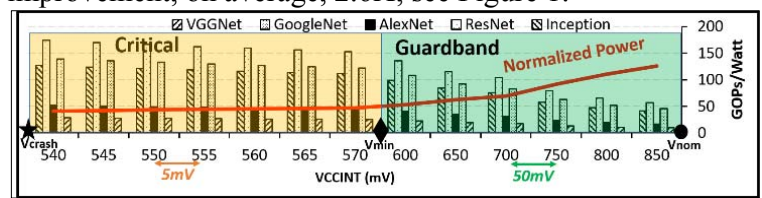

Figure 1: Power-efficiency of FPGA-based DNN via undervolting

With further undervolting below the minimum safe voltage level (i.e., $V_{\min }=570 \mathrm{mV}$ ), the power-efficiency improves by an extra $43 \%$, leading to a total improvement of more than $3 \mathrm{X}$. This additional gain comes with the cost of DNN accuracy collapse, detailed reliability analysis in [10]. To prevent this accuracy loss, we will demonstrate a simultaneous frequency underscaling. The aim is to find $F_{\max }$ at each voltage level, where there is no DNN accuracy loss. This technique reduces the power-efficiency gain from $43 \%$ to $25 \%$, see Table 1 . Further undervolting, FPGA does not operate, and it crashes at $V_{\text {crash }}$ (i.e., $540 \mathrm{mV}$ ).

Tahle 1: Freauencv underscaling in critical voltage region. Best results of each metric are highlighted.

\begin{tabular}{cc|cccc}
\hline $\begin{array}{c}\boldsymbol{V}_{\text {CCINT }} \\
(\mathbf{m V})\end{array}$ & $\begin{array}{c}\boldsymbol{F}_{\max } \\
(\mathbf{M h z})\end{array}$ & $\begin{array}{c}\text { GOPs } \\
(\text { Norm) }\end{array}$ & $\begin{array}{c}\text { Power } \\
(\text { Norm) }\end{array}$ & $\begin{array}{c}\text { GOPs/W } \\
(\mathbf{N o r m})\end{array}$ & $\begin{array}{c}\text { GOPs/J } \\
(\text { Norm) }\end{array}$ \\
\hline $\mathbf{5 7 0}$ & $\mathbf{3 3 3}$ & $\mathbf{1 . 0 0}$ & 1.00 & 1.00 & $\mathbf{1 . 0 0}$ \\
$\mathbf{5 6 0}$ & $\mathbf{2 5 0}$ & 0.83 & 0.84 & 0.99 & 0.75 \\
$\mathbf{5 5 0}$ & $\mathbf{2 5 0}$ & 0.83 & 0.75 & 1.10 & 0.83 \\
$\mathbf{5 4 0}$ & $\mathbf{2 0 0}$ & 0.70 & $\mathbf{0 . 5 6}$ & $\mathbf{1 . 2 5}$ & 0.75 \\
\hline
\end{tabular}

Figure 2 depicts the overall methodological flow. (i) Benchmark: we evaluate undervolting with five commonlyused image classification benchmarks, as detailed them in [9]. This demonstration is based on AlexNet, with eight layers and a large parameter size of 233.2 MB. (ii) Software: For our implementation, we leverage the Xilinx DNNDK tool in the classification phase of DNNs. (iii) Hardware: Our prototype is based on the Xilinx Ultrascale+ ZCU102. We will demonstrate undervolting $V_{C C I N T}$ that is supplying the internal FPGA components.

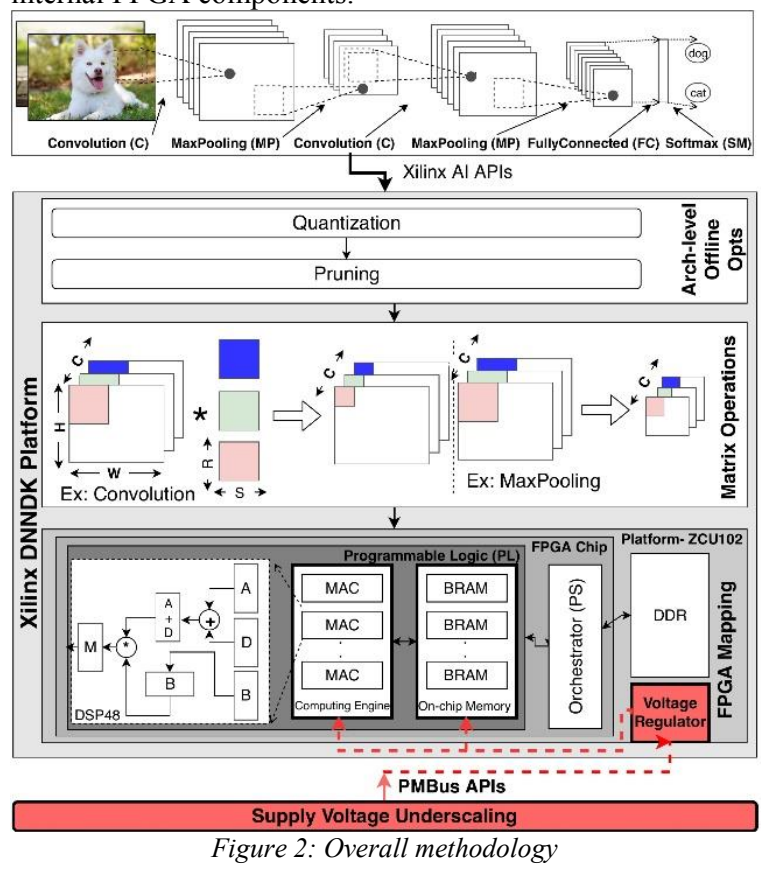

\section{ACKNOWLEDGMENT}

The research leading to these results has received funding from the European Union's Horizon 2020 Programme under the LEGaTO Project (www.legato-project.eu), grant agreement n॰780681.

\section{REFERENCES}

[1] B. Salami, et al., "HATCH: Hash Table Caching in Hardware for Efficient Relational Join on FPGA", in FCCM, 2015.

[2] B. Salami, et al., "AxleDB: A Novel Programmable Query Processing Platform on FPGA", in MICPRO, 2017.

[3] B. Salami, et al., "Accelerating hash-based query processing operations on FPGAs by a hash table caching technique", in CARLA, 2016.

[4] O. Arcas-Abela, "Hardware Acceleration for Query Processing: Leveraging FPGAs, CPUs, and Memory", in CISE, 2016.

[5] B. Salami, et al., "Comprehensive Evaluation of Supply Voltage Underscaling in FPGA On-chip Memories", in MICRO, 2018.

[6] B. Salami, et al., "Fault Characterization Through FPGA Undervolting", in FPL, 2018.

[7] B. Salami, et al., "Evaluating built-in ECC of FPGA on-chip memories for the mitigation of undervolting faults", in PDP, 2019.

[8] K. Givaki, et al., "On the Resilience of Deep Learning for Reducedvoltage FPGAs", in PDP, 2020.

[9] B. Salami, et al., "An Experimental Study of Reduced-Voltage Operation in Modern FPGAs for Neural Network Acceleration" in DSN, 2020.

[10] B. Salami, et al., "On the Resilience of RTL NN Accelerators: Fault characterization and mitigation", in SBAC-PAD, 2018. 